\title{
Erratum to: Noise effects on robust synchronization of a small pacemaker neuronal ensemble via nonlinear controller: electronic circuit design
}

Elie Bertrand Megam Ngouonkadi ${ }^{1} \cdot$ Hilaire Bertrand Fotsin $^{1} \cdot$ Martial Kabong Nono $^{1} \cdot$ Patrick Herve Louodop Fotso ${ }^{1,2}$

Published online: 11 February 2017

(C) Springer Science+Business Media Dordrecht 2017

\section{Erratum to: Cogn Neurodyn (2016) 10:385-404 DOI 10.1007/s11571-016-9393-1}

In the original publication of the article, the Acknowledgement has been missed out. It is given in this erratum.
Acknowledgements P. Louodop acknowledges support by Grant Nr. 2014/13272-1 Sao Paulo Research Foundation (FAPESP).

The online version of the original article can be found under doi:10.1007/s11571-016-9393-1.

Hilaire Bertrand Fotsin

hbfotsin@yahoo.fr

Elie Bertrand Megam Ngouonkadi

elyemegambertrhand@yahoo.fr

Martial Kabong Nono

kabongmartial@yahoo.fr

Patrick Herve Louodop Fotso

louodop@yahoo.fr

1 Laboratory of Electronics and Signal Processing, Department

of Physics, Faculty of Sciences, University of Dschang,

P. O. Box 067, Dschang, Cameroon

2 Instituto de Física Teórica, Universidade Estadual Paulista, UNESP, Rua Dr. Bento Teobaldo Ferraz 271, Bloco II, Barra

Funda, São Paulo 01140-070, Brazil 